\title{
Estratégia de nutrientes para ovinos em distintas sequências de fornecimento alimentar em dieta a base de palma forrageira
}

\author{
Feeding strategy for sheep in different sequences in supplying ingredients based diet of \\ spineless cactus
}

\author{
FOTIUS, Anna Christine Alencar ${ }^{1}$; FERREIRA, Marcelo de Andrade ${ }^{1}$; VÉRAS, \\ Antônia Sherlânea Chaves ${ }^{1}$; SALLA, Luciane Elisete ${ }^{1}$; SOUZA $^{1}$, Andréa Roberto $^{2}$ \\ Duarte Lopes; BISPO ${ }^{2}$, Safira Valença
}

\footnotetext{
${ }^{1}$ Universidade Federal Rural de Pernambuco, Departamento de Zootecnia, Recife, Pernambuco, Brasil.

${ }^{2}$ Universidade Federal da Paraíba, Departamento de Zootecnia, Areia, Paraíba, Brasil.

*Endereço para correspondência: lusalla@yahoo.com.br
}

\section{RESUMO}

O objetivo com este trabalho foi avaliar o efeito da sequência da oferta dos ingredientes da dieta (estratégia alimentar), a base de palma forrageira (Nopalea cochenillifera Salm-Dyck) sobre o consumo, a digestibilidade dos nutrientes, o pH e a concentração de nitrogênio amoniacal do líquido ruminal. Os alimentos foram fornecidos a cinco ovinos de peso médio de $38 \mathrm{~kg}$ nas seguintes sequências: mistura completa $(\mathrm{MC})$ às 7:00h e às 15:00h; concentrado às 7:00h e palma às $8: 00 \mathrm{~h}$ e concentrado às $15: 00 \mathrm{~h}$ e feno de capim tifton às 16:00h (CPCF); concentrado às 7:00h e feno de capim tifton às $8: 00 \mathrm{~h}$ e concentrado às $15: 00 \mathrm{~h}$ e palma às 16:00h ( $\mathrm{CFCP})$; palma às $7: 00 \mathrm{~h}$ e concentrado às $8: 00 \mathrm{~h}$ e feno de capim tifton às 15:00h e concentrado às 16:00h (PCFC); feno de capim tifton às $7: 00 \mathrm{~h}$ e concentrado às $8: 00 \mathrm{~h}$ e palma às $15: 00 \mathrm{~h}$ e concentrado às $16: 00 \mathrm{~h}$ (FCPC). Foi adotado o delineamento quadrado latino $5 \mathrm{X} 5$, com cinco tratamentos, cinco animais e cinco períodos. Os consumos dos nutrientes não foram influenciados pelas sequências. A digestibilidade da matéria seca (MS), matéria orgânica (MO), nutrientes digestíveis totais (NDT), fibra em detergente neutro (FDN) e carboidratos totais (CHOT) diferiram entre os tratamentos. Os valores de $\mathrm{pH}$ ruminal e as concentrações de nitrogênio amoniacal foram influenciados pelos horários de coletas nas sequências FCPC e CFCP. Todas as estratégias de alimentação podem ser utilizadas para alimentação de ovinos, exceto mistura completa.

Palavras-chave: consumo de nutrientes, mistura completa, nitrogênio amoniacal, $\mathrm{pH}$ ruminal

\section{SUMMARY}

The objective of this work was to evaluate the effect of the sequence of the supply of ingredients (feeding strategy), based diet forage cactus (Nopalea cochenillifera Salm Dyck) on intake, digestibility of nutrients, $\mathrm{pH}$ and ammonia concentration in the rumen fluid. The ingredients were provided five sheep of nondescript breeding, averaging $38,5 \mathrm{~kg}$, gifted with rumen cannule the following sequences: 1 - (TMR, total mixed ration) at 7:00 and 15:00 h; $2-(\mathrm{C}$, concentrate $)$ at 7:00 $\mathrm{h},(\mathrm{SC}$, spineless cactus) at 8:00 h, (C) at 15:00 $\mathrm{h}$ and $(\mathrm{TGH}$, tifton grass hay) at 16:00 h; $3-(\mathrm{C})$ at 7:00 h, (TGH) at 8:00 h, (C) at 15:00 h and (SC) at $16: 00 \mathrm{~h} ; 4-(\mathrm{SC})$ at 7:00 h, (C) at 8:00 h, (TGH) at 15:00 and (C) at 16:00 $\mathrm{h}$ and 5$(\mathrm{TGH})$ at 7:00 h, (C) at 8:00 h, (SC) at 15:00 h and $(\mathrm{C})$ at 16:00 $\mathrm{h}$. Were distributed into a $5 \times 5$ Latin Square with five treatments, five animals and five periods. The nutrient intake were not influenced by sequences The digestibility of dry matter (DM), organic matter(OM), total digestible nutrients (TDN), neutral detergent fiber (NDF) and total carbohydrates differ between treatments. The ruminal $\mathrm{pH}$ and ammonia nitrogen were influenced by the times in collections sequences $\mathrm{TGH} / \mathrm{C} / \mathrm{SC} / \mathrm{TGH}$ and $\mathrm{C} / \mathrm{TGH} / \mathrm{C} / \mathrm{SC}$. All feeding strategies can be used to feed sheep, except for total mixed ration.

Keywords: ammonia nitrogen, complete mixing, nutrient intake, ruminal $\mathrm{pH}$ 


\section{INTRODUÇ̃̃̃O}

A palma forrageira é considerada um importante nutriente para ruminantes, em regiões semiáridas, excelente fonte de energia e água. Porém, apresenta baixos teores de fibra em detergente neutro, em torno de 25\% (FERREIRA et al., 2009). O teor de fibra em detergente neutro fisicamente efetiva (FDNfe) é semelhante ao dos concentrados, ou seja, valores abaixo do recomendado para manutenção da ruminação (15\% do FDN) (BISPO et al., 2007). Assim, baixo teor de FDNfe poderá diminuir a ruminação e salivação, quando em grandes proporções na dieta, sendo necessária a associação da palma com um alimento fibroso (SALEM \& SMITH, 2008). Em altas proporções na dieta de ruminantes pode levar a redução no consumo de matéria seca, pelo elevado teor de umidade e de carboidratos não fibrosos, acarretando distúrbios digestivos (VIEIRA et al., 2008). Estes efeitos, associados à diminuição concomitante do $\mathrm{pH}$ ruminal (abaixo de 6,2), pode inibir a atividade celulolítica, deprimir a ingestão da forragem e afetar o aproveitamento dos nutrientes da ração (KOZLOSK et al., 2006). Para evitar eventuais problemas digestivos, opta-se por fornecer a palma associada a fontes de fibra e concentrados, na forma de mistura completa, ou o fornecimento de concentrado separado (PESSOA et al., 2005). Quando todos os ingredientes da dieta são consumidos na forma de $\mathrm{MC}$, ocorre fermentação ruminal mais uniforme, melhorando a utilização dos nutrientes, em decorrência da redução da variação na composição da dieta e seleção dos ingredientes oferecidos (NRC, 2007).

Outra opção seria o fornecimento dos ingredientes separados, em diferentes sequências e horários. $\mathrm{Na}$ prática, dificilmente se fornece a palma como alimento misturado ao concentrado, e sim é oferecido separado dos demais ingredientes da ração (PESSOA et al., 2004; VILELA et al., 2010) e como consequência poderão ocorrer mudanças bruscas no ambiente ruminal. Desconhecem-se os potenciais benefícios da oferta de alimentos separados para maximização do consumo e digestão dos nutrientes, já que o fracionamento do fornecimento dos alimentos em vários horários resultaria em várias refeições durante o dia e estimularia o aumento do consumo de nutrientes.

Objetivou-se avaliar os efeitos de diferentes sequências de fornecimento dos ingredientes da dieta à base de palma forrageira, sobre consumo, digestibilidade, $\mathrm{pH}$ e nitrogênio amoniacal em ovinos.

\section{MATERIAL E MÉTODOS}

O experimento foi realizado no Departamento de Zootecnia da Universidade Federal Rural de Pernambuco. Foram utilizados cinco ovinos machos sem padrão racial definido (SPRD), dotados de cânula ruminal, com peso médio inicial de $38,5 \mathrm{~kg}$, alojados em gaiolas metabólicas providas de comedouro e bebedouro individual.

Cada período experimental teve duração de 21 dias, sendo 14 dias de adaptação e sete dias para coleta de amostras e dados. A dieta foi constituída de $31 \%$ de feno de capim-tifton; $40 \%$ de palma miúda (Nopalea cochenillifera SalmDyck) e $29 \%$ de concentrado, composto por $55,7 \%$ de farelo de soja, $37,2 \%$ milho e 4,88 de mistura mineral. A composição nutricional da dieta, uma vez que foram mudadas apenas a sequência de oferta dos alimentos e ingredientes apresentam-se na Tabela 1. 
Rev. Bras. Saúde Prod. Anim., Salvador, v.15, n.2, p.504-516 abr./jun., 2014 http://www.rbspa.ufba.br ISSN 15199940

Tabela 1. Composição nutricional dos alimentos e da dieta completa

\begin{tabular}{lcrcrr}
\hline Composição química & $\begin{array}{c}\text { Mistura } \\
\text { completa }\end{array}$ & Palma & $\begin{array}{c}\text { Feno de } \\
\text { capim tifton }\end{array}$ & $\begin{array}{r}\text { Farelo } \\
\text { de soja }\end{array}$ & $\begin{array}{r}\text { Milho } \\
\text { moído }\end{array}$ \\
\hline Matéria Seca (\%MN) & 33,43 & 17,10 & 91,76 & 90,11 & 89,14 \\
Matéria Orgânica (\%MS) & 91,08 & 89,47 & 93,68 & 93,39 & 98,40 \\
Proteína Bruta (\%MS) & 12,81 & 2,91 & 5,19 & 53,05 & 8,92 \\
Extrato etéreo (\%MS) & 2,10 & 1,33 & 2,27 & 4,02 & 2,50 \\
Fibra em Detergente Neutro (\%MS) & 37,76 & 20,14 & 78,20 & 28,52 & 11,99 \\
Fibra em Detergente Ácido (\%MS) & 14,55 & 9,52 & 31,84 & 5,47 & 2,26 \\
Carboidratos Totais (\%MS) & 76,17 & 85,23 & 86,21 & 37,86 & 85,23 \\
Carboidratos Não -Fibrosos (\%MS) & 38,41 & 65,09 & 8,01 & 9,01 & 73,44 \\
\hline
\end{tabular}

Os tratamentos experimentais avaliados foram: mistura completa (MC) às 7:00h e às 15:00h; concentrado às 7:00h e palma às $8: 00 \mathrm{~h}$ e concentrado às $15: 00 \mathrm{~h}$ e feno de capim tifton às 16:00h (CPCF); concentrado às 7:00h e feno de capim tifton às $8: 00 \mathrm{~h}$ e concentrado às $15: 00 \mathrm{~h}$ e palma às $16: 00 \mathrm{~h}$ (CFCP); palma às 7:00h e concentrado às $8: 00 \mathrm{~h} \mathrm{e}$ feno de capim tifton às 15:00h e concentrado às 16:00h (PCFC); feno de capim tifton às $7: 00 \mathrm{~h}$ e concentrado às $8: 00 \mathrm{~h}$ e palma às $15: 00 \mathrm{~h}$ e concentrado às 16:00h (FCPC). Os alimentos foram oferecidos de acordo com os tratamentos experimentais, distribuídos em diferentes sequências de fornecimento dos alimentos (Tabela 2).

Tabela 2. Horário de fornecimentos das dietas dos tratamentos experimentais

\begin{tabular}{lcccc}
\hline \multirow{2}{*}{ Tratamento } & \multicolumn{3}{c}{ Horário } \\
\cline { 2 - 5 } Manhã & \multicolumn{3}{c}{ Tarde } \\
\hline 7:00horas & Mistura completa & - & Mistura completa & - \\
CPCF & Concentrado & Palma & Concentrado & Feno de Tifton \\
CFCP & Concentrado & Feno de Tifton & Concentrado & Palma \\
PCFC & Palma & Concentrado & Feno de Tifton & Concentrado \\
FCPC & Feno de Tifton & Concentrado & Palma & Concentrado \\
\hline MC = mistura completa; CFCP = concentrado feno concentrado palma, CPCF = concentrado palma \\
concentrado feno, PCFC = palma concentrado feno concentrado; FCPC = feno concentrado palma \\
concentrado.
\end{tabular}

As dietas foram ajustadas diariamente em função do consumo do dia anterior, com sobras mantidas em torno de $10 \%$ do ofertado. Para estimativa do consumo, diariamente foram registradas as quantidades oferecidas dos alimentos e das sobras e realizadas coletas de amostras dos alimentos e sobras para posteriores análises.

A coleta total de fezes ocorreu por 24 horas, nos setes dias dos períodos de coleta, com amostragens diárias de 10\% do total, após arraçoamento matinal. Ao final de cada período realizou-se uma amostra composta por animal das 
sobras e fezes, sendo estas identificadas, acondicionadas em recipientes plásticos e congeladas $\left(-10^{\circ} \mathrm{C}\right)$ para posteriores análises químicas. As amostras foram secas em estufa de circulação forçada $\left(65^{\circ} \mathrm{C}\right)$, por 72 horas, moídas em moinho tipo Wiley, em peneiras com crivo de $1 \mathrm{~mm}$ de diâmetro.

As análises foram realizadas no Laboratório de Nutrição Animal pertencente ao Departamento de Zootecnia da Universidade Federal Rural de Pernambuco. Para as determinações dos teores de matéria seca (MS), matéria mineral (MM), proteína bruta (PB), extrato etéreo (EE) conforme Silva \& Queiroz (2002). Para a determinação da FDN e FDA utilizou-se autoclave, onde 0,5 g de amostra, acondicionada em sacos de tecidos não- tecidos (TNT), previamente secos e pesados, foram fervidos por uma hora em solução de detergente neutro, utilizando $\alpha$-amilase (VAN SOEST \& ROBERTSON, 1985), lavados com água quente e acetona, secos e pesados, sendo o seu resíduo considerado a FDN. Para a FDA, adotou-se o mesmo procedimento, mas com solução de detergente ácido. Para estimativa dos carboidratos totais (CHO) foi empregada a equação: $100-$ $(\% \mathrm{~PB}+\% \mathrm{EE}+\%$ Cinzas $)$ e para a determinação dos teores de carboidratos não fibrosos: $\mathrm{CNF}=100 \%-(\% \mathrm{~PB}+$ $\% \mathrm{FDN}+\% \mathrm{EE}+\% \mathrm{MM})$, segundo (HALL, 1999).

O coeficiente de digestibilidade aparente dos nutrientes (CDN) foi calculado conforme Silva \& Leão (1979): $\mathrm{CDN}=$ (Nutriente ingerido nutriente excretado/ nutriente ingerido) $\mathrm{x}$ 100. Para estimativa dos nutrientes digestíveis totais (NDT), foi utilizada a equação descrita por Weiss (1999): $\mathrm{NDT}=\mathrm{PBD}+\mathrm{EED}^{*} 2,25+\mathrm{CNFD}$ + FDND, sendo $\mathrm{PBD}=(\mathrm{PB}$ ingerida $\mathrm{PB}$ fezes $), \mathrm{EED}=(\mathrm{EE}$ ingerido $-\mathrm{EE}$ fezes $), \mathrm{CNFD}=(\mathrm{CNF}$ ingeridos $-\mathrm{CNF}$ fezes $)$ e FDND $=($ FDN ingerido - FDN fezes).

As amostras de conteúdo ruminal $( \pm 400 \mathrm{~mL})$ foram colhidas via fistula ruminal em quatro pontos distintos, na região ventral do rúmen e posteriormente homogeneizada. As coletas foram realizadas durante seis dias de cada período, a cada 26 horas, correspondentes a hora zero (imediatamente antes do fornecimento da ração) e a 2; 4; 6; 8 e 10 horas após a primeira alimentação. A digesta foi filtrada e o líquido homogeneizado imediatamente para a mensuração do $\mathrm{pH}$ com potenciômetro digital. Para a determinação do nitrogênio amoniacal $\left(\mathrm{N}-\mathrm{NH}_{3}\right)$ colheu-se uma alíquota de $20 \mathrm{~mL}$ do líquido ruminal, acondicionando-o em frasco de vidro contendo $1 \mathrm{~mL}$ de ácido clorídrico $(6 \mathrm{~N})$ e armazenado a $-10^{\circ} \mathrm{C}$. descongeladas e centrifugadas a $3000 \mathrm{rpm}$ por 15 minutos, conforme técnica descrita por Fenner (1965) e adaptada.

O delineamento utilizado foi o quadrado latino 5X5; cinco tratamentos, cinco animais e cinco períodos. Aplicou-se análise de variância sendo as médias comparadas pelo teste de Tukey, em nível de $5 \%$ de probabilidade. Foi utilizado o sistema de análise estatística e genética-SAEG (UFV, 2007).

\section{RESULTADO E DISCUSSÃO}

Não foi observada diferença significativa $\quad(\mathrm{P}>0,05) \quad$ entre os tratamentos para o consumo médio diário de MS (Tabela 3). Resultado esperado, uma vez que as dietas foram formuladas para atender as exigências de manutenção dos animais (NRC, 2007). Os consumos de MO, PB, EE, FDN, CNF e CHOT não foram influenciados pelos tratamentos, 
provavelmente devido à ingestão similar de matéria seca da dieta.

Pessoa et al. (2004) avaliaram o fornecimento de $\mathrm{MC}$ e ingredientes separados à vacas leiteiras, e não observaram diferenças no consumo de MS, porém apesar dos ingredientes separados terem sido fornecidos nos mesmos horários, a estratégia alimentar baseada em ingredientes separados pode contribuir para o desbalanceamento da dieta ingerida e redução da produtividade animal. Por outro lado, a possibilidade de seleção do alimento para os animais mantidos sob a estratégia de $\mathrm{MC}$ é bastante reduzida, contribuindo para o equilíbrio na participação dos ingredientes na dieta.

Tabela 3. Consumo de nutrientes em ovinos alimentados com diferentes sequências alimentares

\begin{tabular}{|c|c|c|c|c|c|c|}
\hline \multirow{2}{*}{ Composição química } & \multicolumn{5}{|c|}{ Tratamentos } & \multirow{2}{*}{$\begin{array}{l}\mathrm{CV}^{1} \\
(\%)\end{array}$} \\
\hline & $\mathrm{MC}$ & CFCP & $\mathrm{CPCF}$ & PCFC & FCPC & \\
\hline Matéria Seca (kg/dia) & 1,26 & 1,18 & 1,29 & 1,28 & 1,19 & 11,05 \\
\hline Matéria Seca $(\% \mathrm{PV})$ & 3,35 & 3,09 & 3,42 & 3,36 & 3,10 & 10,60 \\
\hline Matéria Seca $\left(\% \mathrm{PV}^{0,75}\right)$ & 83,03 & 76,93 & 84,69 & 83,50 & 77,24 & 10,72 \\
\hline Matéria Orgânica (kg/dia) & 1,16 & 1,09 & 1,19 & 1,18 & 1,10 & 11,06 \\
\hline Proteína Bruta (kg/dia) & 0,17 & 0,16 & 0,18 & 0,18 & 0,16 & 10,77 \\
\hline Fibra em Detergente Neutro (kg/dia) & 0,44 & 0,40 & 0,45 & 0,46 & 0,42 & 12,88 \\
\hline Fibra em Detergente Neutro (\%PV) & 1,16 & 1,04 & 1,20 & 1,20 & 1,10 & 12,18 \\
\hline Carboidratos totais $(\mathrm{kg} / \mathrm{dia})$ & 0,97 & 0,91 & 0,99 & 0,98 & 0,92 & 11,23 \\
\hline Carboidratos Não- Fibrosos (kg/dia) & 0,54 & 0,50 & 0,55 & 0,54 & 0,49 & 11,67 \\
\hline Extrato Etéreo $(\mathrm{kg} / \mathrm{dia})$ & 0,03 & 0,02 & 0,03 & 0,03 & 0,02 & 11,11 \\
\hline Nutrientes digestíveis totais $(\mathrm{kg} / \mathrm{dia})$ & 0,88 & 0,80 & 0,96 & 0,97 & 0,85 & 13,08 \\
\hline
\end{tabular}

$\mathrm{MC}=$ mistura completa; $\mathrm{CFCP}=$ concentrado feno concentrado palma, $\mathrm{CPCF}=$ concentrado palma concentrado feno, PCFC = palma concentrado feno concentrado; FCPC = feno concentrado palma concentrado. ${ }^{1}$ Coeficiente de variação.

O consumo é o componente que exerce o papel de maior importância na nutrição animal, pois determina o nível de nutrientes ingeridos. Mertens (1994) propôs que o consumo voluntário é regulado por três mecanismos: o fisiológico, cuja regulação é pelo balanço nutricional; o físico, relacionado com a capacidade de distensão do rúmen; e o psicogênico, que envolve o comportamento do animal a fatores inibidores ou estimuladores relacionados ao alimento ou ao ambiente. Dos mecanismos, o fisiológico provavelmente regulou o consumo de MS, pois a ingestão de energia foi similar (Tabela
3), indicando que independente das sequências de fornecimento, os animais ingeriram os nutrientes de forma a atender ao máximo suas demandas nutricionais.

Silva et al. (2005) e Wongnen et al. (2009) observaram que o fornecimento da dieta na forma de mistura completa aumentou o consumo de alimentos pelos animais, embora a espécie animal utilizada tenha sido diferente e as dietas à base de milho. Souza et al. (2010) verificaram que os consumos de MS e FDN aumentaram quando ovelhas receberam dieta com palma, feno e concentrado na forma de mistura 
completa e justificaram o aumento pela aderência do feno e do concentrado à palma na homogeneização da dieta.

Vilela et al. (2010) afirmaram que alimentos fornecidos na forma de mistura completa quando comparados ao fornecimento separados dos alimentos, permite menor consumo de concentrado por evitar a seleção do alimento, diferentemente do que ocorre com alimentos separados, onde o animal consome todo o concentrado de uma única vez, devido sua alta aceitabilidade, não sendo visualizado estes componentes nas sobras. Porém, os resultados observados neste estudo foram distintos dos relatados na literatura e indicam ser possível reduzir custos operacionais para mistura de dieta total (volumoso e concentrado), já que não foi necessário oferecer dietas totalmente homogeneizadas para que os animais apresentassem o adequado consumo de MS e nutrientes.

Foram observadas diferenças significativas entre as médias de digestibilidade da MS, MO, FDN e carboidratos totais $(\mathrm{P}<0,05)$ e nutrientes digestíveis totais (Tabela 4).

Quando foram ofertados palma e concentrado, pela manhã, independentemente da sequência PCFC e CPCF, houve superioridade nas digestibilidades de MS, MO, FDN e CHOT $(\mathrm{P}<0,05)$ em relação aos tratamentos MC e CFCP (Tabela 4). O FCPC apresentou valores de digestibilidade semelhantes a todos os tratamentos $(\mathrm{P}>0,05)$.

Tabela 4. Digestibilidade aparente de nutrientes e valor dos nutrientes digestíveis totais da dieta experimental de ovinos alimentados com diferentes sequências alimentares

\begin{tabular}{lcccccc}
\hline \multirow{2}{*}{ Item } & \multicolumn{5}{c}{ Tratamentos } & $\mathrm{CV}^{1}$ \\
\cline { 2 - 6 } & $\mathrm{MC}$ & CFCP & CPCF & PCFC & FCPC & $(\%)$ \\
\hline Matéria Seca & $70,30^{\mathrm{b}}$ & $69,68^{\mathrm{b}}$ & $74,70^{\mathrm{a}}$ & $75,41^{\mathrm{a}}$ & $71,66^{\mathrm{ab}}$ & 3,00 \\
Matéria Orgânica & $73,17^{\mathrm{b}}$ & $72,48^{\mathrm{b}}$ & $77,44^{\mathrm{a}}$ & $77,55^{\mathrm{a}}$ & $74,71^{\mathrm{ab}}$ & 2,67 \\
Fibra em Detergente Neutro & $49,89^{\mathrm{b}}$ & $46,72^{\mathrm{b}}$ & $59,76^{\mathrm{a}}$ & $62,70^{\mathrm{a}}$ & $53,86^{\mathrm{ab}}$ & 8,95 \\
Carboidratos totais & $72,78^{\mathrm{b}}$ & $72,18^{\mathrm{b}}$ & $77,85^{\mathrm{a}}$ & $77,26^{\mathrm{a}}$ & $74,47^{\mathrm{ab}}$ & 2,83 \\
\hline \multicolumn{7}{c}{ Valor energético } \\
\hline Nutrientes digestíveis totais & $69,83^{\mathrm{b}}$ & $67,73^{\mathrm{b}}$ & $75,44^{\mathrm{a}}$ & $75,16^{\mathrm{a}}$ & $71,11^{\mathrm{ab}}$ & 3,32 \\
\hline
\end{tabular}

$\mathrm{MC}=$ mistura completa; $\mathrm{CFCP}=$ concentrado feno concentrado palma, $\mathrm{CPCF}=$ concentrado palma concentrado feno, PCFC = palma concentrado feno concentrado; FCPC $=$ feno concentrado palma concentrado.

${ }^{1}$ Coeficiente de variação, valores seguidos por letras minúsculas diferentes na linha diferem $(\mathrm{P}<0,05)$ entre si pelo teste de Tukey.

Os valores superiores dos tratamentos PCFC e CPCF podem estar associados às características nutricionais da palma forrageira e seu aproveitamento em dietas com concentrado. Moreira et al. (2009) mencionaram que dietas com elevada concentração de CNF reduzem o $\mathrm{pH}$ ruminal. Isto pode induzir acidose, causando redução de consumo e a absorção de nutrientes deficiente, tanto quanto deprimir o desempenho do animal. Entretanto, a palma forrageira não favorece a produção de lactato e propionato e sim de acetato, ácido graxo volátil relevante na manutenção do $\mathrm{pH}$ ruminal. Por apresentar teores 
consideráveis de pectina, carboidrato estrutural de fermentação peculiar, que gera grande quantidade de energia por unidade de tempo, como ocorre com o amido e açúcares, porém com fermentação acética, que caracteriza a celulose e a hemicelulose, proporcionando, provavelmente, melhor padrão de fermentação ruminal.

Por sua vez, a palma, devido sua estrutura promove mastigação mais intensa e com isso um aumento da produção de saliva, favorecendo a estabilidade do $\mathrm{pH}$. Como a sequência PCFC também apresentou valor de digestibilidade superior aos tratamentos MC e CFCP. Neste caso, a manutenção do $\mathrm{pH}$ para melhorias na digestibilidade provavelmente está associado ao fato dos animais terem recebido feno da sequência seguinte, à tarde, sendo este um volumoso que provavelmente contribuiu para estabilidade do $\mathrm{pH}$ ruminal, por também estimular a produção de saliva pela ruminação, que pode ter resultado em aumento da atividade microbiana com fermentação mais uniforme e consequentemente em melhorias na utilização dos nutrientes.
Da mesma forma, estes fatores podem ter contribuído para semelhança observada na digestibilidade dos nutrientes entre o tratamento FCPC e os tratamentos que receberam palma $\mathrm{e}$ concentrado, pela manhã. Independente da sequência de fornecimento, o valor médio de $\mathrm{pH}$ ruminal mostrou-se em valores adequados para manutenção da eficiência da fermentação ao longo do dia (6,33). Embora o tratamento CFCP tenha apresentado o mesmo comportamento em termos de estabilidade de $\mathrm{pH}$, não foi observada alteração na digestibilidade dos nutrientes.

A mistura completa e a sequência concentrado e feno (CFCP), na alimentação da manhã, apresentaram menor digestibilidade (Tabela 5), resultados contraditórios aos do NRC (2007), que afirma que a MC promove ótimo equilíbrio de nutrientes para os microrganismos e fermentação ruminal mais uniforme, melhor utilização dos nutrientes, pois reduz a variação na composição da dieta e seleção dos ingredientes ofertados.

Tabela 5. Valores de $\mathrm{pH}$ do líquido ruminal de ovinos alimentados com diferentes sequências alimentares

\begin{tabular}{|c|c|c|c|c|c|c|}
\hline \multirow{2}{*}{$\mathrm{pH}$} & \multicolumn{5}{|c|}{ Tratamentos } & \multirow{2}{*}{$\mathrm{CV}^{1}(\%)$} \\
\hline & $\mathrm{MC}$ & CFCP & $\mathrm{CPCF}$ & PCFC & FCPC & \\
\hline 7 horas & 6,64 & 6,28 & 6,40 & 6,70 & 6,43 & 3,39 \\
\hline 9 horas & $6,04^{\mathrm{bc}}$ & $6,33^{\mathrm{ab}}$ & $6,06^{\mathrm{bc}}$ & $5,97^{\mathrm{c}}$ & $6,40^{a}$ & 2,58 \\
\hline 11 horas & $5,90^{\mathrm{b}}$ & $6,23^{a}$ & $5,92^{\mathrm{b}}$ & $5,83^{\mathrm{b}}$ & $6,43^{a}$ & 2,00 \\
\hline 13 horas & $6,14^{\mathrm{ab}}$ & $6,36^{\mathrm{a}}$ & $5,78^{\mathrm{b}}$ & $5,85 \mathrm{~b}$ & $6,47^{\mathrm{a}}$ & 3,27 \\
\hline 15 horas & $6,12^{\mathrm{b}}$ & $6,36^{\mathrm{ab}}$ & $6,02^{\mathrm{b}}$ & $6,12^{\mathrm{b}}$ & $6,55^{\mathrm{a}}$ & 3,13 \\
\hline 17 horas & 5,64 & 6,00 & 5,73 & 5,97 & 5,75 & 4,76 \\
\hline Média & $6,07^{\mathrm{b}}$ & $6,27^{\mathrm{a}}$ & $5,99^{\mathrm{b}}$ & $6,07^{\mathrm{b}}$ & $6,33^{a}$ & 1,43 \\
\hline \multicolumn{7}{|c|}{$\begin{array}{l}\mathrm{MC}=\text { mistura completa; } \mathrm{CFCP}=\text { concentrado feno concentrado palma, } \mathrm{CPCF}=\text { concentrado palma } \\
\text { concentrado feno, PCFC }=\text { palma concentrado feno concentrado; FCPC }=\text { feno concentrado palma } \\
\text { concentrado. } \\
{ }^{1} \text { Coeficiente de variação, valores seguidos por letras minúsculas diferentes na linha diferem }(\mathrm{P}<0,05)\end{array}$} \\
\hline
\end{tabular}


$\mathrm{O}$ valor de digestibilidade do tratamento FCPC não diferiu dos demais tratamentos $(\mathrm{P}>0,05)$, indicando que o mesmo proporcionou padrão de fermentação similar às demais sequências estudadas, independente do período de fornecimento. Logo, permite o produtor escolher o volumoso a ser utilizado (palma ou feno) pela manhã ou à tarde, conforme a disponibilidade de mão de obra para triturá-lo.

Foram observadas diferenças significativas nos valores de $\mathrm{pH}$ entre os tratamentos $(\mathrm{P}>0,05)$. $\mathrm{O} \mathrm{pH}$ também foi influenciado $(\mathrm{P}<0,05)$ pelas diferentes horas de aferição, com exceção daquele observado às $7 \mathrm{~h}$ e $17 \mathrm{~h}$ (Tabela 5). $\mathrm{O} \mathrm{pH}$ é uma função dependente do tipo de alimentação e seus efeitos sobre a produção de saliva, relacionado à quantidade de fibra na dieta e à ruminação, produção e absorção de AGV, nível de consumo de ração e de troca de bicarbonato através do epitélio ruminal (MERTENS, 1994).

A similaridade das médias de $\mathrm{pH}$ observadas nas coletas realizadas às 7 horas era esperada, pois a aferição foi realizada antes da alimentação. Logo, houve tempo disponível durante o período da noite para ruminação e produção de saliva para que houvesse a estabilidade do $\mathrm{pH}$, pela manhã, já que neste cenário os animais já tinham consumido toda a dieta do dia antes deste.

Entretanto, o pH observado às $9,11,13$ e 15 horas e o $\mathrm{pH}$ médio sofreram influência dos tratamentos $(\mathrm{P}<0,05)$. As sequências onde o feno foi fornecido pela manhã (FCPC e CFCP) foram as que proporcionaram os maiores valores de $\mathrm{pH}$. Provavelmente o perfil nutricional do volumoso tenha influenciado os valores, já que a fermentação do feno, rico em fibra em detergente neutro, é responsável principalmente pela produção de acetato (ácido graxo volátil que não promove abaixamento de $\mathrm{pH}$ ruminal) e por estimular maior tempo de ruminação durante a noite, pode ter promovido maior fluxo de saliva que teria favorecido a estabilização do $\mathrm{pH}$ (MOTTA et al., 2010).

Os tratamentos em que a palma e o concentrado foram fornecidos pela manhã (CPCF e PCFC), independentemente da sequência, apresentaram menores valores de $\mathrm{pH} \quad(\mathrm{P}>0,05)$. Isto possivelmente ocorreu devido a presença de concentrado e maior produção de ácidos graxos voláteis resultante da fermentação dos carboidratos não fibrosos, que potencializa uma queda do $\mathrm{pH}$ ruminal (HOMEM JÚNIOR et al., 2010). Segundo os autores, dietas com concentrado reduzem o $\mathrm{pH}$ ruminal, podem induzir à acidose e acarretar redução do consumo e absorção deficiente dos nutrientes em função da elevação na concentração de ácido lático. Entretanto, apesar do $\mathrm{pH}$ ruminal ter sido alterado (Figura 1), o consumo de nutrientes no presente trabalho não foi deprimido (Tabela 3), indicando que a combinação dos ingredientes influenciou mais o consumo do que as sequências dos alimentos.

Entretanto, é possível observar que independentemente do tratamento, o pH sempre se manteve acima de 5,64. Segundo Valadares Filho \& Pina (2006), quando o pH fica entre 5,0 e 5,5 muitos microrganismos ruminais cessam o seu crescimento, apesar de conseguirem sobreviver mesmo em altas concentrações de $\mathrm{H}^{+}$. Contudo, o pH baixo pode retardar a adesão dos microrganismos à celulose, em decorrência da deficiência de compostos que aumentam a adesão, semelhantes ao bicarbonato, ou ao aumento dos que inibem a adesão como o amido solúvel. 


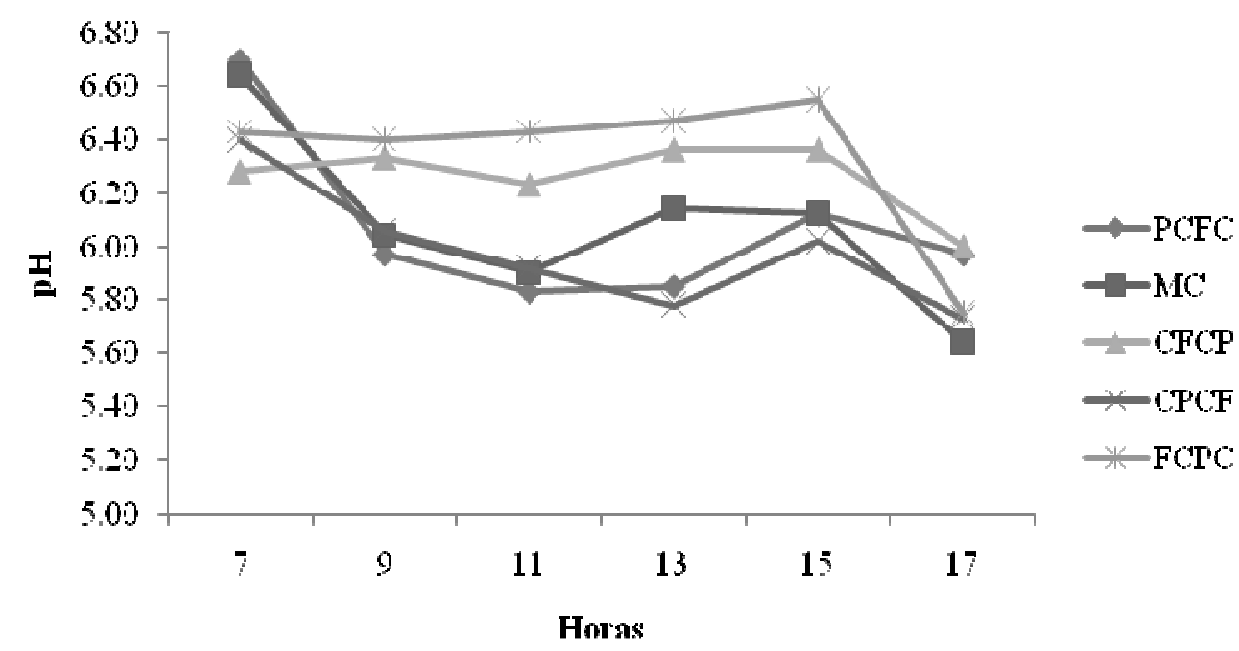

$\mathrm{PCFC}=$ palma concentrado feno concentrado, $\mathrm{MC}=$ mistura completa, $\mathrm{CFCP}=$ concentrado feno concentrado palma, $\mathrm{CPCF}=$ concentrado palma concentrado feno, $\mathrm{FCPC}=$ feno concentrado concentrado palma.

Figura 1. Valores de $\mathrm{pH}$ do líquido ruminal de ovinos alimentados com diferentes sequências alimentares

Quando a dieta foi fornecida na forma de mistura completa, o $\mathrm{pH}$ ruminal apresentou menor valor em relação às sequências CFCP e FCPC $(\mathrm{P}>0,05)$. Provavelmente pela seleção de alimentos, possivelmente houve seleção pelo ingrediente mais palatável (concentrado).

Embora observadas algumas alterações nos valores de $\mathrm{pH}$, a média de cada tratamento foi ideal para o funcionamento normal do rúmen, contudo, o comportamento do $\mathrm{pH}$ durante as dez horas de coleta (7 às $17 \mathrm{~h}$ ) mostra que as diferentes formas de fornecimento da dieta influenciaram diretamente o $\mathrm{pH}$. Portanto, a média geral de $\mathrm{pH}$ pode não ser conclusiva à respeito das diferentes sequências de fornecimento, uma vez que os tratamentos apresentaram valores de $\mathrm{pH}$ distintos nos horários devido ao perfil de fermentação de cada alimento das dietas.

A concentração de nitrogênio amoniacal foi influenciada nos diferentes horários de coleta, exceto para aquela obtida às $7 \mathrm{~h}$ da manhã $(\mathrm{P}<0,05)$. O NH3 foi influenciado significativamente pelos tratamentos $(\mathrm{P}<0,05)$ nos horários de 9 , 11, 13, e 15 horas (Tabela 6).

Quando o concentrado e o feno foram ofertados na alimentação, pela manhã (CFCP e FCPC), a concentração de N$\mathrm{NH} 3$ no líquido ruminal foi superior aos tratamentos MC, CPFC e PCFC (Tabela 6), devido a falta de sincronia entre energia e proteína, principalmente pela elevada fração não-degradável da FDN de capim-Tifton 85 e a composição do concentrado, com maior participação de farelo de soja.

As concentrações de $\mathrm{N}^{-\mathrm{NH}_{3}}$ (Figura 2) foram superiores quando $\mathrm{o}$ feno $\mathrm{e}$ concentrado foram ofertados pela manhã (FCPC e CFCP), provavelmente, pela falta de sincronização entre a energia e nitrogênio, uma vez que a baixa degradabilidade da fibra do feno não promoveria liberação de energia suficiente para a utilização do nitrogênio. 
Rev. Bras. Saúde Prod. Anim., Salvador, v.15, n.2, p.504-516 abr./jun., 2014 http://www.rbspa.ufba.br ISSN 15199940

Nas sequências palma e concentrado (CPCF e PCFC) fornecidos pela manhã, verificou-se os menores valores de nitrogênio amoniacal $(\mathrm{P}>0,05)$. Uma vez que a palma possui CNF rapidamente fermentáveis, possivelmente, houve sincronização entre fornecimento de nitrogênio e de energia, levando a maior utilização do nitrogênio amoniacal e com isso as concentrações dos mesmos foram menores, corroborando com Chapaval et al. (2008). Comportamento semelhante observou-se para MC. Devido à oferta concomitante total dos alimentos, proporcionando a utilização do nitrogênio com os CNF rapidamente fermentáveis da palma, em que os grupamentos carbonados foram supridos por alimentos que continham alta concentração de carboidratos solúveis e taxa de fermentação semelhante ao farelo de soja, corroborando com Menezes et al. (2009).

Tabela 6 Nitrogênio amoniacal $\left(\mathrm{N}_{-} \mathrm{NH}_{3} \mathrm{mg} / 100 \mathrm{ml}\right)$ do líquido ruminal de ovinos alimentados com diferentes sequências alimentares

\begin{tabular}{lcccccc}
\hline \multirow{2}{*}{$\mathrm{N}-\mathrm{NH}_{3} \mathrm{mg} / 100 \mathrm{ml}$} & $\mathrm{MC}$ & $\mathrm{CFCP}$ & $\mathrm{CPCF}$ & PCFC & FCPC & $\begin{array}{c}\mathrm{CV}^{1} \\
(\%)\end{array}$ \\
\cline { 2 - 5 } 7 Horas & 14,33 & 11,10 & 15,43 & 13,23 & 12,71 & 30,51 \\
9 Horas & $11,02^{\mathrm{c}}$ & $15,21^{\mathrm{ab}}$ & $13,30 \mathrm{~b}^{\mathrm{c}}$ & $10,73^{\mathrm{c}}$ & $17,12^{\mathrm{a}}$ & 10,90 \\
11 Horas & $8,23^{\mathrm{b}}$ & $13,60^{\mathrm{a}}$ & $6,22^{\mathrm{b}}$ & $8,09^{\mathrm{b}}$ & $16,10^{\mathrm{a}}$ & 23,25 \\
13 Horas & $9,77^{\mathrm{b}}$ & $16,90^{\mathrm{a}}$ & $8,23^{\mathrm{b}}$ & $7,50^{\mathrm{b}}$ & $20,06^{\mathrm{a}}$ & 16,30 \\
15 Horas & $11,61^{\mathrm{b}}$ & $22,49^{\mathrm{a}}$ & $10,58^{\mathrm{b}}$ & $7,28^{\mathrm{b}}$ & $18,30^{\mathrm{a}}$ & 15,60 \\
17 Horas & $13,52^{\mathrm{ab}}$ & $18,30^{\mathrm{a}}$ & $11,17^{\mathrm{b}}$ & $12,79^{\mathrm{ab}}$ & $16,54^{\mathrm{ab}}$ & 22,12 \\
\hline Média & $11,42^{\mathrm{b}}$ & $16,27^{\mathrm{a}}$ & $10,82^{\mathrm{b}}$ & $9,93^{\mathrm{b}}$ & $16,80^{\mathrm{a}}$ & 8,55 \\
\hline
\end{tabular}

${ }^{1}$ Coeficiente de variação, valores seguidos por letras minúsculas diferentes na mesma linha diferem $(\mathrm{P}<0,05)$ entre si pelo teste de Tukey.

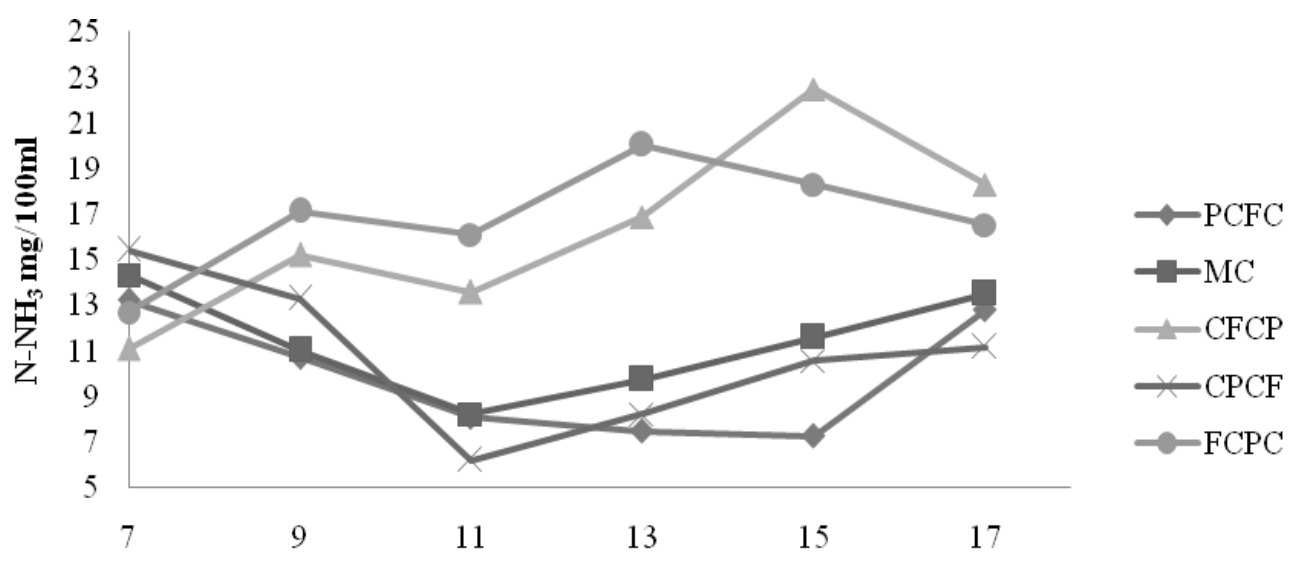

$\mathrm{PCFC}=$ palma concentrado feno concentrado, $\mathrm{MC}=$ mistura completa, $\mathrm{CFCP}=$ concentrado feno concentrado palma, $\mathrm{CPCF}=$ concentrado palma concentrado feno, $\mathrm{FCPC}=$ feno concentrado concentrado palma.

Figura 2. Nitrogênio amoniacal $\left(\mathrm{N}-\mathrm{NH}_{3} \mathrm{mg} / 100 \mathrm{ml}\right)$ do líquido ruminal de ovinos alimentados com diferentes sequências alimentares 
Rev. Bras. Saúde Prod. Anim., Salvador, v.15, n.2, p.504-516 abr./jun., 2014 http://www.rbspa.ufba.br ISSN 15199940

As diferentes sequências de oferta dos alimentos não afetaram o consumo de nutrientes. Contudo, a sequência de palma e concentrado pela manhã, independentemente do horário de fornecimento dos ingredientes, promove melhor digestibilidade dos nutrientes, menor concentração de nitrogênio amoniacal e $\mathrm{pH}$ ruminal adequado para aproveitamento da energia, conforme indicado pelos resultados superiores de nutrientes digestíveis totais.

\section{REFERÊNCIAS}

BISPO, S.V.; FERREIRA, M.A.; VÉRAS, A.S.C.; ATISTA, A.M.V.; PESSOA, R.A.S.; BLEUEL, P.M.P. Palma forrageira em substituição ao feno de capim-elefante. Efeito sobre consumo, digestibilidade e características de fermentação ruminal em ovinos. Revista Brasileira de Zootecnia, v.36, n.6, p.1902-1909 2007.

CHAPAVAL, L.; MELOTTI, L.; ROSSI JÚNIOR, P.; OLIVINDO, C.S.; REGO, J.P.A. Relação volumoso concentrado sobre as concentrações ruminais de amônia, $\mathrm{pH}$ e ácidos graxos voláteis em vacas leiteiras mestiças. Revista Brasileira de Saúde e Produção Animal [on line], v.9, n.1, p.18-28, 2008.

FENNER, H. Methods for determining total volatile base in rumen fluid by steam distillation. Journal of Dairy Science, v.48, n.3, p.249-251, 1965.

FERREIRA, M. de A.; SILVA, R.R.; RAMOS, A.O. Síntese de proteína microbiana e concentrações de uréia em vacas alimentadas com dietas à base de palma Forrageira e diferentes volumosos. Revista Brasileira de Zootecnia, v.38, n.1, p.159-165, 2009.
HALL, M.B.; HOOVER, W.H.; JENNINGS, J.P.; WEBSTER, T.K.M. A Method for partitioning neutral detergent soluble carbohydrates. Journal of the Science of Food Agriculture, v.79, n.15, p.2079-2086, 1999.

HOMEM JÚNIOR, A.C.; EZEQUIEL, J.M.B.; FÁVARO, V.R.; OLIVEIRA, P.S.N.; D’AUREA, A.P.; SANTOS, V.C.; GONÇALVES, J.S. Fermentação ruminal de ovinos alimentados com alto concentrado e grãos de girassol ou gordura protegida. Arquivos Brasileiro Medicina Veterinária e Zootecnia, v.62, n.1, p.144-153, 2010.

KOZLOSKI, G.V.; NETTO, D.P.; BONNECARRÈRE SANCHEZ, L.M.; LIMA, L.D.; CADORIN JÚNIOR, R. L.; FIORENTINI, G.; HARTER, C.J. Nutritional value of diets based on a low-quality grass hay supplemented or not with urea and levels of cassava meal. African Journal of Agricultural Research, v.1, n.3, p.38-46, 2006.

MENEZES, D.R.; ARAÚJO, G.G.L.; SOCORRO E.P.; OLIVEIRA R.L.; BAGALDO A.R.; SILVA, T.M.; PEREIRA, LGR. Níveis de uréia em dietas contendo co-produto de vitivinícolas e palma forrageira para ovinos Santa Inês. Arquivos Brasileiro de Medicina Veterinária e Zootecnia, v.61, n.3, p.662-667, 2009.

MERTENS, D.R. Regulation of forage intake. In: NATIONAL CONFERENCE ON FORAGE QUALITY. EVALUATION AND UTILIZATION, 1994, Lincoln. Proceedings... Lincoln: University of Nebraska. 1994. p.450-493. 
Rev. Bras. Saúde Prod. Anim., Salvador, v.15, n.2, p.504-516 abr./jun., 2014 http://www.rbspa.ufba.br ISSN 15199940

MOREIRA, P.C.; MENDONÇA, A.C.; MARTINS, A.F.; WASCHECK, R.C.; SOUZA, P.R.; DUTRA, A.R.; GRANDSIRE, C.; REZENDE, P.L.P.; CARDOSO, J.R.; BENETTI, E.J.; SILVA, M.S.B. Avaliação do $\mathrm{pH}$ do fluido ruminal de vacas leiteiras.

Estudos, v.36, n.11/12, p.1201-1218, 2009.

MOTTA, M.F.; VILELA, D.; SANTOS, G.T.; ELYAS, A.C.W.; LOPES, F.C.F.; VERNEQUE, R.S.; PAIVA, P.C.A.; PINTO NETO, A.P. Parâmetros ruminais de vacas leiteiras mantidas em pastagem tropical.

Archivos de Zootecnia, v.59, n.226, p.217-224, 2010.

NATIONAL RESEARCH COUNCIL NRC. Nutrients requeriments of small ruminants. Washington, DC, 2007. $362 p$.

PESSOA, R.A.S.F.; ERREIRA, M.A.; LIMA L.E.; LIRA, M.A.; VÉRAS, A.S.C.; SILVA, A.E.V.N.; SOSA, M.Y.; AZEVEDO, M.; MIRANDA, K.F.; SILVA, F.M.; MELO, A.A.S.; LÓPEZ, O.R.M. Desempenho de vacas leiteiras submetidas a diferentes estratégias alimentares. Revista Archivos de Zootecnia, v.53, n.203, p.309-320, 2004.

PESSOA, R.A.S; FERREIRA, M.A.; LIMA, L.E.A.Vacas leiteiras submetidas a diferentes estratégias alimentares. Digestibilidade e balanço de energia. Acta Scientiaurum.

Animal Sciences, v.27, n.2, p.269-276, 2005.

SALEM, H. B.; SMITH, T. Feeding strategies to increase small ruminant production in dry environments. Small Ruminant Research, v.77, n.2, p.174194, 2008.
SILVA, D. J.; QUEIROZ, A.C. Análise de Alimentos: métodos químicos e biológicos. 3 ed. Viçosa: Universidade Federal de Viçosa, 2002. 235p.

SILVA, A.E.V.N.; GUIM, A.; FERREIRA, M.A.; LIMA, L.E.; PESSOA, R. A.S.; SOSA, M.Y. Estratégia alimentar para dieta baseada em palma forrageira sobre o desempenho e digestibilidade em vacas em final de lactação, Acta Scientiarum Animal Science, v.27, n.2, p.269-276, 2005.

SILVA, J.F.C.; LEÃO, M.I. Fundamentos da nutrição de ruminantes. Piracicaba, SP, ed. Livroceres, p.380, 1979.

SOUZA, C.M.S.; MEDEIROS, A.N.; FURTADO, D.A.; BATISTA,A.M.V.; PIMENTA FILHO, E.C.; SILVA, D.S. Desempenho de ovelhas nativas em confinamento recebendo palmaforrageira na dieta na região do semiárido nordestino. Revista Brasileira de Zootecnia, v.39, n.5, p.1146-1153, 2010.

UNIVERSIDADE FEDERAL DE VIÇOSA - UFV. SAEG - Sistema de análise estatística e genética. Versão 8.0. Viçosa, $M G, 2007$. 150p.

VALADARES FILHO, S. de C.; PINA, D. dos S. Fermentação Ruminal. In: BERCHIELLI, T.T.; PIRES, A.V.; OLIVEIRA, S.G. (Eds.). Nutrição de Ruminantes. Jaboticabal: FUNEP, 2006. p.151-182,

Van SOEST, P.J; RORBERTSON, J.B. A Laboratory Manual for Animal Science. Ithaca, New York: Cornell University, 1985. 
Rev. Bras. Saúde Prod. Anim., Salvador, v.15, n.2, p.504-516 abr./jun., 2014 http://www.rbspa.ufba.br ISSN 15199940

VIEIRA, E.L.; BATISTA, A.M.V.; GUIM, A.; CARVALHO, F.F.R.; NASCIMENTO, A.C.O.; ARAÚJO, R.F.S. da S. ; MUSTAFA, A.F. Effects of hay inclusion on intake, total tract nutrient utilization and ruminal fermentation of goats fed spineless cactus (Opuntiaficus-indica Mill) based diets. Animal Feed Science and Technology, v 141, n.3-4, p.199-208, 2008.

VILELA, M.S.; FERREIRA, M.A.; AZEVEDO, M.; MODESTO, E.C.; FARIAS, I.; GUIMARAES, A.V.; BISPO, S.V. Effect of processing and feeding strategy of the spineless cactus (Opuntiafícus-indica Mill.) for lactating cows: Ingestive behavior. Applied Animal Behavior Science, v.125, n.1, p.1-8, 2010.
WEISS, W.P. Energy prediction equations for ruminant feeds. In: CORNELL NUTRITION CONFERENCE FOR FEED MANUFACTURERS, 61., 1999, Ithaca. Proceedings... Ithaca: Cornell University, 1999. p.176-185.

WONGNEN, KONGWEHA, K.; NAMSAEN, N.; GUNUN, P.; YUANGKLANG, C. Effects of fermented total mixed ration and cracked cottonseed on milk yield and milk composition in dairy cows, AsianAustralasian Journal of Animal Sciences, v.22, n.12, p.1625-1632, 2009.

Data de recebimento: 30/12/2013

Data de aprovação: 26/06/2014 